\title{
A prediction about the age of thick discs as a function of the stellar mass of the host galaxy
}

\author{
S. Comerón ${ }^{1,2}$ \\ 1 Departamento de Astrofísica, Universidad de La Laguna, 38200 La Laguna, Tenerife, Spain \\ e-mail: seb.comeron@gmail.com \\ 2 Instituto de Astrofísica de Canarias, 38205 La Laguna, Tenerife, Spain
}

Received 18 December 2020 / Accepted 12 January 2021

\section{ABSTRACT}

\begin{abstract}
One of the suggested thick disc formation mechanisms is that they were born quickly and in situ from a turbulent clumpy disc. Subsequently, thin discs formed slowly within them from leftovers of the turbulent phase and from material accreted through cold flows and minor mergers. In this Letter, I propose an observational test to verify this hypothesis. By combining thick disc and total stellar masses of edge-on galaxies with galaxy stellar mass functions calculated in the redshift range of $z \leq 3.0$, I derived a positive correlation between the age of the youngest stars in thick discs and the stellar mass of the host galaxy; galaxies with a present-day stellar mass of $\mathcal{M}_{\star}(z=0)<10^{10} \mathcal{M}_{\odot}$ have thick disc stars as young as 4-6 Gyr, whereas the youngest stars in the thick discs of Milky-Way-like galaxies are $\sim 10 \mathrm{Gyr}$ old. I tested this prediction against the scarcely available thick disc age estimates, all of them are from galaxies with $\mathcal{M}_{\star}(z=0) \gtrsim 10^{10} \mathcal{M}_{\odot}$, and I find that field spiral galaxies seem to follow the expectation. On the other hand, my derivation predicts ages that are too low for the thick discs in lenticular galaxies, indicating a fast early evolution for S0 galaxies. I propose the idea of conclusively testing whether thick discs formed quickly and in situ by obtaining the ages of thick discs in field galaxies with masses of $\mathcal{M}_{\star}(z=0) \sim 10^{9.5} \mathcal{M}_{\odot}$ and by checking whether they contain $\sim 5$ Gyr-old stars.
\end{abstract}

Key words. galaxies: evolution - galaxies: spiral - galaxies: structure

\section{Introduction}

Thick discs in galaxies are the low surface brightness and large scale-height counterparts of the canonical thin discs. Thick disc stars are older than those of the thin discs (e.g., Bensby et al. 2005 for the Milky Way, and Yoachim \& Dalcanton 2008; Comerón et al. 2015; Pinna et al. 2019a for other galaxies) and they comprise at least several tens of percent of the baryons in discs (e.g., Reid 2005; Fuhrmann 2008; Fuhrmann et al. 2012; Snaith et al. 2014 for the Milky Way, and Yoachim \& Dalcanton 2006; Comerón et al. 2011, 2018 for other galaxies). Hence, thick discs are key to understand how early galaxies evolved.

The origin of thick discs is controversial. The proposed formation mechanisms cover the four corners of the diagram that summarises the processes of galactic evolution in Kormendy \& Kennicutt (2004). The position of a mechanism in this diagram is characterised by two parameters: whether it is internally- or environment-driven, and whether the process is fast (of the order of a dynamical timescale) or secular. In this framework, the possible thick disc formation mechanisms are as follows: (1) external fast processes, where the thick disc is a consequence of the merger of gas-rich galaxies during the initial galaxy assembly process (Brook et al. 2004; Martig et al. 2014), (2) internal fast processes where the thick disc is born thick due to the turbulent and clumpy nature of the first discs (Elmegreen \& Elmegreen 2006; Bournaud et al. 2009; Comerón et al. 2014), (3) external secular processes where the thick disc is made of stars stripped from infalling satellites (Abadi et al. 2003) and by the disc dynamical heating caused by satellites (Qu et al. 2011), and (4) internal secular processes, where the thick disc is caused by dynamical heating due to disc overdensities (Villumsen 1985) and/or the radial migration of stars (Schönrich \& Binney 2009; Roškar et al. 2013).

Several of the abovementioned mechanisms might be acting concurrently. For example, simulations have shown that the combination of internally and externally driven secular heating can produce realistic thick discs (Minchev et al. 2015; García de la Cruz et al. 2020). Another example is the fact that the thick discs in three S0 galaxies in the Fornax cluster show traces of a significant minority of accreted stars on top of a population that was formed in situ (Pinna et al. 2019a,b). It is also possible that galaxies with different masses have different thick disc formation paths (Comerón et al. 2012).

In this Letter, I present a prediction about the age of the youngest stars of thick discs as a function of the stellar mass of the host galaxy if the main formation mechanism is internal and fast. The confrontation between this prediction and forthcoming spectroscopically-derived thick disc ages will make it possible to confirm or reject the scenario put to the test.

I assume that the formation of thick disc stars is completed before the thin disc starts forming (Elmegreen \& Elmegreen 2006; Bournaud et al. 2009) and that the thick disc and the classical bulge form simultaneously (Comerón et al. 2014). In this framework, the first discs were clumpy and with a large star formation rate, roughly ten times larger than that in the present day. Because the star formation scale height scales with the specific star formation rate (Comerón et al. 2014), these discs were thick. Due to dynamical friction, some of the clumps spiralled inwards and formed bulges. The thick disc and the classical bulge are collectively called the dynamically hot components. 


\section{Derivation}

Here I derive the age of the youngest stars in thick discs (and classical bulges) as a function of the host stellar mass for thick discs which form quickly and in situ. Given a galaxy with a present-day stellar mass $\mathcal{M}_{\star}(z=0)$, two elements are required to do so: (1) the mass of the dynamically hot components, $\mathcal{M}_{\mathrm{h}}$, and (2) the evolution with redshift of the total stellar mass, $\mathcal{M}_{\star}(z)$.

The first point can be addressed by resorting to the decompositions of Spitzer Survey of Stellar Structure in Galaxies $\left(S^{4} G\right.$; Sheth et al. 2010) edge-on galaxies by Comerón et al. (2018), which provide the masses of the thin discs, the thick discs, and the classical bulges. The risk of confusion between pseudobulges and classical bulges was minimised by assigning the light of flattened components to the disc (usually the thin disc). Figure 1 displays the hot component mass, $\log \left(\mathcal{M}_{\mathrm{h}} / \mathcal{M}_{\odot}\right)$, as a function of the total stellar mass, $\log \left(\mathcal{M}_{\star}(z=0) / \mathcal{M}_{\odot}\right)$, for the 124 galaxies with a fitted thick disc in Comerón et al. (2018). A linear regression to the scatter plot gives

$\log \left(\mathcal{M}_{\mathrm{h}} / \mathcal{M}_{\odot}\right)=0.489+0.9161 \log \left(\mathcal{M}_{\star}(z=0) / \mathcal{M}_{\odot}\right)$.

To find how the stellar mass of galaxies evolves with time, I followed the method proposed by van Dokkum et al. (2013). For a given stellar mass at present, $\mathcal{M}_{\star}(z=0)$, I find the progenitors at a given redshift, $z$, by assuming that the comoving density does not vary with time. This approach is valid as long as galaxies conserve their mass rank order, which is true within 0.15 dex in the simulations by Leja et al. (2013). In practice, this is done by integrating galaxy stellar mass functions at different redshifts, $\phi(\mathcal{M}, z)$, to find the mass, $\mathcal{M}_{\star}(z)$, above which the cumulative comoving density is equal to that for the present-day stellar mass $\mathcal{M}_{\star}(z=0)$

$$
\begin{aligned}
\rho\left(\mathcal{M}>\mathcal{M}_{\star}(z=0)\right) & =\int_{\mathcal{M}_{\star}(z=0)}^{\infty} \phi(\mathcal{M}, z=0) \mathrm{d} \mathcal{M} \\
& =\int_{\mathcal{M}_{\star}(z)}^{\infty} \phi(\mathcal{M}, z) \mathrm{d} \mathcal{M}=\rho\left(\mathcal{M}>\mathcal{M}_{\star}(z)\right) .
\end{aligned}
$$

Leja et al. (2020) compiled data from galaxies in the redshift range of $0.2 \leq z \leq 3.0$ and constructed a continuity model to describe $\phi(\mathcal{M}, z)$ at an arbitrary redshift within the studied range. Their model was built using all the masses and redshifts of the galaxies in their sample simultaneously, without using any sort of binning in either mass or redshift. I computed the mass functions with a python code provided by Leja et al. (2020) and used the mass functions and Eq. (2) to derive the stellar mass evolution of galaxies with $\mathcal{M}_{\star}(z=0)$ between $10^{9}$ and $10^{11.2} \mathcal{M}_{\odot}$. The evolution at $z<0.2$ was calculated with extrapolated stellar mass functions. The resulting stellar mass evolution estimates are presented in Fig. 2.

Under the assumption that the thick disc and the classical bulge form before the thin disc, Eq. (1) and the information in Fig. 2 can be combined to obtain, for each present-day stellar mass, $\mathcal{M}_{\star}(z=0)$, the redshift when the thick disc formation ended, that is the redshift for which

$\mathcal{M}_{\mathrm{h}}=\mathcal{M}_{\star}(z)$.

These redshifts can be transformed into an age, $\tau$, so they can be compared with the observed thick disc ages. For consistency with Leja et al. (2020), I assume the WMAP9 cosmology (Hinshaw et al. 2013). The resulting age of the youngest stars in thick discs as a function of the host stellar mass is shown in Fig. 3.



Fig. 1. Scatter plot of the sum of the masses of the thick disc and the classical bulge (dynamically hot components; $\mathcal{M}_{\mathrm{h}}$ ) versus the total stellar mass of the galaxy, $\mathcal{M}_{\star}(z=0)$, for the 124 galaxies with a fitted thick disc in Comerón et al. (2018). The black continuous line indicates the linear fit to all the data, whereas the continuous grey line indicates the fit to the data points corresponding to galaxies in the Virgo and Fornax clusters only. The dashed grey line indicates a one-to-one relation.

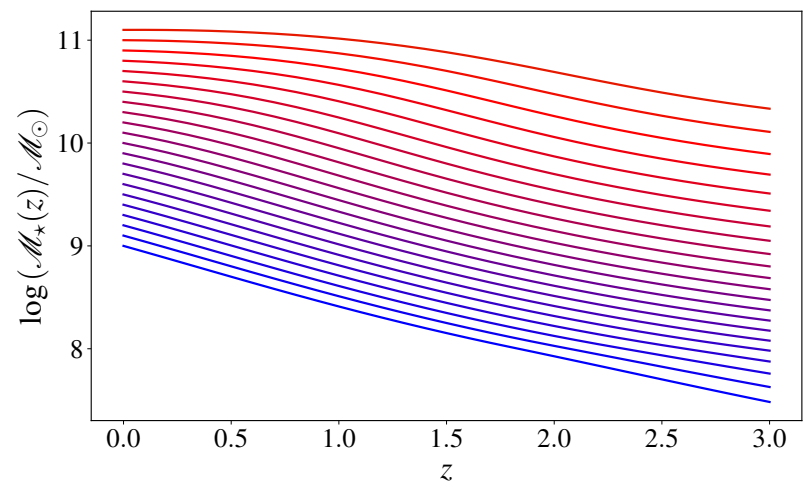

Fig. 2. Stellar mass as a function of redshift for galaxies with presentday stellar masses between $10^{9}$ and $10^{11.2} \mathcal{M}_{\odot}$ (in 0.1 dex intervals) calculated using the continuity model from Leja et al. (2020).

I estimated confidence intervals with a Monte-Carlo approach by redoing the linear fit in Eq. (1) from bootstrapped data points drawn from Fig. 1 and combining the new fit with a new version of the curves in Fig. 2 calculated from the Leja et al. (2020) model with the parameters randomised assuming one- $\sigma$ Gaussian uncertainties. I carried out 1000 repetitions to estimate the one- $\sigma$ uncertainty bands in the figures.

Figure 3 shows that massive galaxies are expected to have very old thick discs with ages around 10 Gyr. Lower-mass galaxies, notably those with stellar masses below $10^{10} \mathcal{M}_{\odot}$, are expected to have thick discs that stopped forming stars much later, between 4 and 6 Gyr ago. The curve shown in Fig. 3 is closely matched by a quadratic function in the mass range of interest

$\tau($ in Gyr $)=104-22.15 \log \left(\mathcal{M}_{\star}(z=0)\right)+1.237\left(\log \left(\mathcal{M}_{\star}(z=0)\right)\right)^{2}$. 


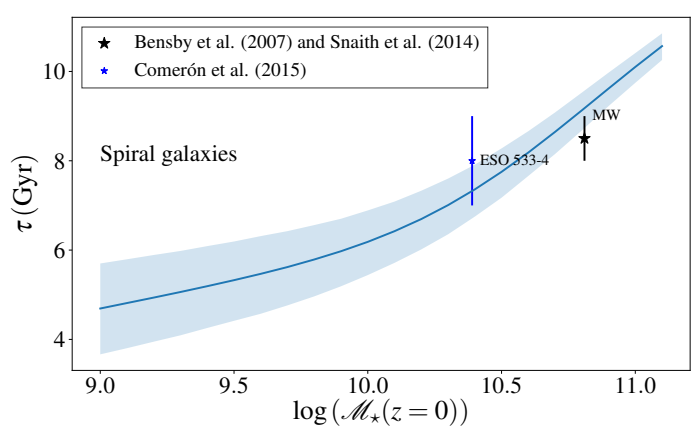

Fig. 3. Predicted age of the youngest stars in thick discs as a function of the total present-day stellar mass of the host. The error bands correspond to one- $\sigma$ confidence intervals. The symbols correspond to spiral galaxies with thick disc stellar ages and masses reported in the literature.

\section{Comparison with observations}

In this section, the prediction that thick discs are older in the most massive galaxies is confronted with thick disc ages that were obtained spectroscopically for a few galaxies with thick disc stellar masses derived in Comerón et al. (2018). Strictly speaking, the prediction concerns the age of the youngest stars in the thick disc. However, from an observational perspective, the difference is small because the luminosity of a galaxy component is dominated by its youngest stars.

The observational data and thick disc ages have been obtained by different groups. Hence, the data are heterogeneous, both in their acquisition and processing, as explained below. The recovery of star formation histories from spectra is an ill-posed inverse problem, which might result in large uncertainties in the estimated ages. Furthermore, bursty star formation is smoothed by regularisation. As a consequence, if the thick discs formed in a short burst, the recovered star formation history would be an artificially broadened peak causing an underestimation of the age of the youngest thick disc stars.

I find that the two non-lenticular galaxies, the Milky Way and ESO 533-4, approximately fall in the relation derived in Sect. 2 (Fig. 3). On the other hand, lenticular galaxies do not follow the relation (Fig. 4).

ESO 533-4 (Comerón et al. 2015). The data were obtained with the optical integral field spectrograph (IFS) VIMOS at the VLT. The thick disc age was estimated with pPXF (Cappellari \& Emsellem 2004). In order to account for a possible excessive regularisation-induced smoothing, I estimated the age of the newest stars in the thick disc to be 7-9 Gyr by finding the interval between which $90 \%$ and $99 \%$ of thick disc stars were formed according to the fit.

NGC 4111, NGC 4710, and NGC 5422 (Kasparova et al. 2016). The data were obtained with the single-slit spectrographs SCORPIO and SCORPIO-2 at BTA. The ages of the thick discs were estimated with NBURSTS (Chilingarian et al. 2007a,b). This yielded 5 Gyr for NGC 4111, 4-5 Gyr for NGC 4710, and 8-11 Gyr for NGC 5422. In the latter two galaxies, however, the slit was placed in regions where the light is dominated by the thin disc according to Comerón et al. (2018). In the case of NGC 4111, the galaxy is thick disc-dominated everywhere at $3.6 \mu \mathrm{m}$ (Comerón et al. 2018), but the slit was placed only 400 pc above the midplane, in a region where the thin disc has a significant contribution at $3.6 \mu \mathrm{m}$, which is probably even larger in the optical. Therefore, the estimated thick disc ages for these galaxies might be significantly biased downwards by the thin disc.

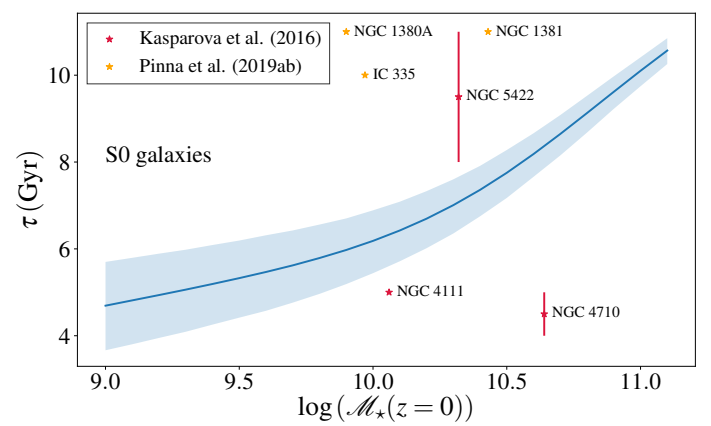

Fig. 4. Same as Fig. 3, but with data points corresponding to S0 galaxies.

IC 335, NGC 1380A, and NGC 1381 (Pinna et al. 2019a,b). The observations of these S0 galaxies in the Fornax cluster were made with the IFS MUSE at the VLT. The authors obtained thick disc star formation histories with pPXF and found that the thick discs have a small fraction of accreted stars that are younger than the in situ population. The accreted stars are distinguishable from those born in situ because of their high $[\mathrm{Mg} / \mathrm{Fe}]$. As this Letter is about in situ thick discs, the age that has been adopted is that of the youngest age bin that is not $[\mathrm{Mg} / \mathrm{Fe}]$-enhanced (10 Gyr for IC 335, $11 \mathrm{Gyr}$ for NGC 1380A, and $11 \mathrm{Gyr}$ for NGC 1381). If some residual in situ thick disc star formation remained by then, the age of the youngest stars in the thick disc would be overestimated.

Data for the Milky Way are also included. I used an age range of 8-9 Gyr (Bensby et al. 2007) for the youngest thick disc stars, which is compatible with an age of $9 \mathrm{Gyr}$ found by Snaith et al. (2014). I also used a stellar mass of $\mathcal{M}_{\star}(z=0)=5 \times 10^{10} \mathcal{M}_{\odot}$ (McMillan 2011).

\section{Discussion: Potential different evolution for the thick discs in spiral and $\mathbf{S O}$ galaxies}

The derivation in Sect. 2 shows that if thick discs form quickly and in situ, those in low-mass galaxies form slower. This is the consequence of two concurrent factors: (1) the smaller the total stellar mass of a galaxy, the larger the relative thick disc mass (Yoachim \& Dalcanton 2006; Comerón et al. 2011, 2012, 2014, 2018); and (2) downsizing, which is the process by which largemass galaxies form faster than small-mass ones (Cowie et al. 1996). These two factors are demonstrated in Figs. 1 and 2, respectively.

Based on the scarce observational evidence, the predicted trend between the age of the youngest stars in a thick disc and the total stellar mass is not followed by S0 galaxies. On the other hand, the two spiral galaxies for which we have data seem to follow it. Assuming that the literature data are representative of the whole population, it is important to determine what the implications are.

Two of the three lenticular galaxies in Kasparova et al. (2016) have thick disc ages that fall well below the expectation (Fig. 4), although most likely, they are measuring a population with a significant thin disc contribution. On the other hand, some lenticular galaxies - including the three galaxies in the Fornax cluster - have ages that are above those predicted. This might be due to a difference in evolutionary paths between isolated galaxies and those in clusters. Sil'chenko et al. (2012) and Comerón et al. (2016) propose that, for at least some S0 galaxies, the evolution when entering a cluster is greatly accelerated (perhaps almost as in a monolithic collapse). Regarding the galaxies discussed 
in Sect. 3, this is clearly the case for NGC 1381, where the thin and the thick discs show no age difference (Pinna et al. 2019b). Because the thin disc is more metal-rich than the thick disc, it is likely that the thin disc is slightly younger, but the difference in age cannot be resolved (see the case of ESO 243-49 in Comerón et al. 2016). On the other hand, IC 335 and NGC 1380A have thin discs whose mean age is noticeably younger than the thick disc, perhaps indicating a 'traditional' evolution where star formation is not quenched by the environment, or indicating that the galaxy has been rejuvenated by accreting fresh material, as suggested by Katkov et al. (2019).

The possibility of two evolutionary paths distinguishing galaxies in the field and at least some lenticular galaxies in clusters is intriguing. Furthermore, it could undermine some of the premises under which this study was done: (1) that all the galaxies fall into a single $\log \mathcal{M}_{\mathrm{h}}$ versus $\log \mathcal{M}_{\star}(z=0)$ relation, and (2) that the evolution of the stellar mass function does not depend on the galaxy type. Point (1) was tested by redoing the fit in Fig. 1 with the 31 galaxies belonging to either the Virgo or the Fornax clusters (continuous grey line). The differences between the fit including cluster galaxies and that with all galaxies are insignificant. This indicates that the differences between spiral and lenticular galaxies are probably related to different mass growth rates.

If the mass evolution differs significantly between cluster and field galaxies, the relationship between the age of the youngest stars in the thick disc and the stellar mass $\mathcal{M}_{\star}(z=0)$ presented in Eq. (4) is a weighted average of two relations. The first one is a relation for cluster disc galaxies or, at least, for some cluster lenticulars. This relation would be much shallower than that in Eq. (4), and probably nearly flat at $\tau \sim 10 \mathrm{Gyr}$ for the range of stellar masses sampled by the observations in Sect. 3, that is $\mathcal{M}_{\star}(z=0) \gtrsim 10^{10} \mathcal{M}_{\odot}$. The second relation is for field disc galaxies. This relation would fall below that presented in Eq. (4) in the mass range of $\mathcal{M}_{\star}(z=0) \gtrsim 10^{10} \mathcal{M}_{\odot}$. Because of the many assumptions made in the derivation of Eq. (4), and the fact that field and cluster galaxies probably have significantly different evolutionary paths, a scatter larger than that presented by the error bands in Fig. 3 is expected.

If the thick discs hosted in field galaxies with stellar masses of $\mathcal{M}_{\star}(z=0) \sim 10^{9.5} \mathcal{M}_{\odot}$ are significantly younger than thick discs in Milky-Way-like galaxies (e.g., ages of $5 \mathrm{Gyr}$ versus $\sim 10$ Gyr for the Milky Way), it would be a good indication that dynamically hot components form before the thin disc does. Additional observations with world-class spectrographs such as MUSE could indeed provide such constraints.

\section{Conclusions}

In order to shed light on the origin of thick discs, I propose a test to verify one of the proposed formation mechanisms. The test concerns the hypothesis that thick discs formed quickly and in situ from turbulent clumpy discs before the thin disc formed within them (Elmegreen \& Elmegreen 2006; Bournaud et al. 2009; Comerón et al. 2014).

By combining the thick disc and the total stellar masses of edge-on galaxies in the $S^{4} G$ from Comerón et al. (2018) and a model allowing me to calculate galaxy stellar mass functions in the redshift range of $0.2 \leq z \leq 3.0$ (Leja et al. 2020), I derived a relationship between the age of the youngest stars in the thick discs and total stellar mass of the host. I thus predict that the age of the youngest thick disc stars increases with the host stellar mass: as young as $4-6 \mathrm{Gyr}$ in galaxies with a total stellar mass below $\mathcal{M}_{\star}(z=0) \sim 10^{10}$, versus $\sim 10 \mathrm{Gyr}$ in Milky-Way-like galaxies.
The scarce available observational data indicate that spiral field galaxies (including the Milky Way) seem to follow the expectation. However, lenticular galaxies in clusters have thick disc stars that are older than predicted, perhaps indicating a very fast early evolution as suggested by Sil'chenko et al. (2012) and Comerón et al. (2016).

To strengthen the tentative evidence that field galaxies formed their thick discs in situ and quickly at high redshift, further observations are required, especially for the unexplored mass range of $\mathcal{M}_{\star}(z=0)<10^{10} \mathcal{M}_{\odot}$. We might expect to find that galaxies with a total stellar mass of $\mathcal{M}_{\star}(z=0) \sim 10^{9.5} \mathcal{M}_{\odot}$ have thick disc stars as young as $\sim 5 \mathrm{Gyr}$.

Acknowledgements. I thank the anonymous referee for comments that have helped to improve this Letter. I thank Dr. Simón Díaz-García for useful discussions and Prof. Johan H. Knapen for revising the manuscript. I acknowledge support from the State Research Agency (AEI-MCINN) of the Spanish Ministry of Science and Innovation under the grant 'The structure and evolution of galaxies and their central regions' with reference PID2019-105602GBI00/10.13039/501100011033.

\section{References}

Abadi, M. G., Navarro, J. F., Steinmetz, M., \& Eke, V. R. 2003, ApJ, 597, 21 Bensby, T., Feltzing, S., Lundström, I., \& Ilyin, I. 2005, A\&A, 433, 185 Bensby, T., Zenn, A. R., Oey, M. S., \& Feltzing, S. 2007, ApJ, 663, L13 Bournaud, F., Elmegreen, B. G., \& Martig, M. 2009, ApJ, 707, L1

Brook, C. B., Kawata, D., Gibson, B. K., \& Freeman, K. C. 2004, ApJ, 612, 894 Cappellari, M., \& Emsellem, E. 2004, PASP, 116, 138

Chilingarian, I., Prugniel, P., Sil'Chenko, O., \& Koleva, M. 2007a, in Stellar Populations as Building Blocks of Galaxies, eds. A. Vazdekis, \& R. Peletier, 241,175

Chilingarian, I. V., Prugniel, P., Sil'Chenko, O. K., \& Afanasiev, V. L. 2007b, MNRAS, 376, 1033

Comerón, S., Elmegreen, B. G., Knapen, J. H., et al. 2011, ApJ, 741, 28

Comerón, S., Elmegreen, B. G., Salo, H., et al. 2012, ApJ, 759, 98

Comerón, S., Elmegreen, B. G., Salo, H., et al. 2014, A\&A, 571, A58

Comerón, S., Salo, H., Janz, J., Laurikainen, E., \& Yoachim, P. 2015, A\&A, 584, A34

Comerón, S., Salo, H., Peletier, R. F., \& Mentz, J. 2016, A\&A, 593, L6

Comerón, S., Salo, H., \& Knapen, J. H. 2018, A\&A, 610, A5

Cowie, L. L., Songaila, A., Hu, E. M., \& Cohen, J. G. 1996, AJ, 112, 839

Elmegreen, B. G., \& Elmegreen, D. M. 2006, ApJ, 650, 644

Fuhrmann, K. 2008, MNRAS, 384, 173

Fuhrmann, K., Chini, R., Hoffmeister, V. H., \& Bernkopf, J. 2012, MNRAS, 420, 1423

García de la Cruz, J., Martig, M., Minchev, I., \& James, P. 2020, MNRAS, submitted [arXiv:2012.02741]

Hinshaw, G., Larson, D., Komatsu, E., et al. 2013, ApJS, 208, 19

Kasparova, A. V., Katkov, I. Y., Chilingarian, I. V., et al. 2016, MNRAS, 460, L89

Katkov, I. Y., Kniazev, A. Y., Kasparova, A. V., \& Sil'chenko, O. K. 2019, MNRAS, 483, 2413

Kormendy, J., \& Kennicutt, R. C., Jr. 2004, ARA\&A, 42, 603

Leja, J., van Dokkum, P., \& Franx, M. 2013, ApJ, 766, 33

Leja, J., Speagle, J. S., Johnson, B. D., et al. 2020, ApJ, 893, 111

Martig, M., Minchev, I., \& Flynn, C. 2014, MNRAS, 443, 2452

McMillan, P. J. 2011, MNRAS, 414, 2446

Minchev, I., Martig, M., Streich, D., et al. 2015, ApJ, 804, L9

Pinna, F., Falcón-Barroso, J., Martig, M., et al. 2019a, A\&A, 625, A95

Pinna, F., Falcón-Barroso, J., Martig, M., et al. 2019b, A\&A, 623, A19

Qu, Y., Di Matteo, P., Lehnert, M. D., \& van Driel, W. 2011, A\&A, 530, A10

Reid, I. N. 2005, ARA\&A, 43, 247

Roškar, R., Debattista, V. P., \& Loebman, S. R. 2013, MNRAS, 433, 976

Schönrich, R., \& Binney, J. 2009, MNRAS, 396, 203

Sheth, K., Regan, M., Hinz, J. L., et al. 2010, PASP, 122, 1397

Sil'chenko, O. K., Proshina, I. S., Shulga, A. P., \& Koposov, S. E. 2012, MNRAS, 427,790

Snaith, O. N., Haywood, M., Di Matteo, P., et al. 2014, ApJ, 781, L31 van Dokkum, P. G., Leja, J., Nelson, E. J., et al. 2013, ApJ, 771, L35

Villumsen, J. V. 1985, ApJ, 290, 75

Yoachim, P., \& Dalcanton, J. J. 2006, AJ, 131, 226

Yoachim, P., \& Dalcanton, J. J. 2008, ApJ, 683, 707 